\title{
The Impact of Power Structures on Supply Chain Enterprises with Carbon Emission Reduction Decisions
}

\author{
Fan $\operatorname{Dandan}^{1} \& \mathrm{Xu} \mathrm{Qi}^{1}$ \\ ${ }^{1}$ Glorious Sun School of Business and Management, Donghua University, Shanghai, China \\ Correspondence: Fan Dandan, Glorious Sun School of Business and Management, Donghua University, \\ Shanghai, China. E-mail: sunshinemarcle@163.com.
}

Received: March 12, 2018

doi:10.5539/ijbm.v13n8p246
Accepted: June 26, 2018

Online Published: June 30, 2018

URL: https://doi.org/10.5539/ijbm.v13n8p246

\begin{abstract}
This paper focuses on analyzing the impact of power structures of supply chain enterprises on their carbon emission reduction decisions. First, three game models (Nash, Manufacturer Stackelberg and Retailer Stackelberg) are constructed according to members' different bargaining power, respectively. Then, the optimal carbon emission reduction decisions and profits of supply chain enterprises in different game models are solved and compared. The research results show that supply chain enterprises have the lowest carbon emission reduction rate in Nash game, and their corresponding profits are the least. As for Stackelberg game, the carbon emission reduction rate as a leader is greater than that as a follower, but the profit as a leader is less than that as a follower. The total profit of the entire supply chain system in Manufacturer Stackelberg model is always greater than that in Retailer Stackelberg model.
\end{abstract}

Keywords: supply chain, power structures, carbon emission reduction, game theory

\section{Introduction}

In the era of increasing global warming, reducing carbon dioxide emission has become a consensus of international communities. According to the Paris Agreement reached in 2015, all parties would participate in the global response to climate change in an "independent contribution" approach. The developed countries will continue to take the lead in reducing emissions and strengthen financial, technological and capacity-building support for the developing countries to help them mitigate and adapt to climate change. In this context, research on supply chain carbon reduction decisions has great practical significance.

There is a mature literature stream about low-carbon supply chains which covers carbon management in low-carbon supply chains, the impact of policies (e.g., carbon tax, carbon trading, and carbon subsidy) on the performance of low-carbon supply chains. There are also some papers concerning low-carbon supply chain design. For example, Hua et al. (2011) investigated how firms manage carbon footprints in inventory management under the carbon emission trading mechanism. Hsu et al. (2013) utilized the Decision-making Trial and Evaluation Laboratory approach to realize carbon management in green supply chains which could improve the overall performance of suppliers. Zakeri et al. (2015) compared the supply chain performance under carbon tax and carbon emissions trading policy schemes via an analytical supply chain planning model. Yu and Han (2017) studied the impact of carbon tax on carbon emission and retail price in a two-echelon supply chain. Yi and $\mathrm{Li}$ (2018) studied effects of government subsidies and carbon tax on the decisions of supply chain members and found that government should levy carbon taxes against manufacturers according to their pollution levels. Chaabane et al. (2011) presented a comprehensive methodology to address sustainable supply chain design problems where carbon emissions and total logistics costs, etc. were considered in the design phase, and the results showed how emission trading market can be used to reduce the carbon dioxide abatement cost. Elhedhli and Merrick (2012) considered a supply chain network design problem that takes CO2 emissions into account, and the test results indicated that considering emission costs could change the optimal configuration of the supply chain. Zhao et al. (2012) used evolutionary game theory to analyze the strategies selected by manufacturers to reduce carbon emissions.

In recent years, an increasing number of studies focus on carbon emission reduction decisions for low-carbon supply chains from different perspectives. He et al. (2015) studied an emission-dependent dyadic fashion supply chain consisting of a supplier and a manufacturer, by exploiting Stackelberg games in four models depending on 
channel structure, comparing their emission reduction efficiencies and profitability for each pair of settings. Du et al. (2015) adopted a novel emission-sensitive demand function, introduced an emission-sensitive cost function, and then investigated the different emission reduction strategies of each member in centralized and decentralized supply chains. Wang et al. (2016) focused on a dyadic supply chain with a manufacturer and a retailer, developed a game model to study the carbon emission reduction decision of the manufacturer within the retailer-dominant case and the power-balanced case, respectively. Ji et al. (2017) focused on the emission reduction behaviors of the chain members in both the retail-channel and dual-channel cases using the Stackelberg game model.

Although the above studies also explore the carbon emission reduction decision, note that Wang et al. (2016) focuses on the decision of the manufacturer and do not take into consideration the decisions of all members of supply chain, as we do in this paper. Moreover, compared with our research, He et al. (2015), Du et al. (2015), and Ji et al. (2017) does not analyze the impact of the bargaining power of the manufacturer and the retailer on their decisions. Specifically, based on the power structures of supply chain enterprises, this paper constructs three game models: Nash, Manufacturer Stackelberg and Retailer Stackelberg. The optimal carbon emission reduction decisions of supply chain enterprises in different game models are discussed and compared in order to guide the practice of different types of enterprises.

\section{Model Description and Assumptions}

Consider a two-tier supply chain system consisting of one manufacturer and one retailer. The manufacturer emits carbon in the process of producing raw materials, while the retailer does that in the distribution process (transportation and sales). Supply chain enterprises can reduce carbon emissions through investment on carbon abatement technology. The greater the carbon abatement investment, the better the carbon emission reduction effects. However, more investment would impair profits of supply chain enterprises, so these enterprises might reduce their efforts in carbon emission reduction. Therefore, it is very important for enterprises to make the optimal carbon reduction decisions.

Power structures of supply chain enterprises vary. When they are evenly matched, the game between the manufacturer and the retailer is a Nash game. When one of them is a leader and the other is a follower, the game between the two parties is a Stackelberg game. Different game behaviors are bound to affect the carbon emission reduction decisions of supply chain enterprises. Therefore, this paper mainly discusses the impact of the different game models on the optimal carbon emission reduction decisions of supply chain enterprises:

Nash (N): In this scenario, the manufacturer and the retailer move simultaneously when deciding on their own carbon emission reduction rate.

Manufacturer Stackelberg (M): In this scenario, the manufacturer, as the Stackelberg leader, moves first to set its carbon emission reduction rate. The retailer, as the follower, decides its carbon emission reduction rate based on the manufacturer's decision.

Retailer Stackelberg (R): In this scenario, the retailer, as the Stackelberg leader, moves first to set its carbon emission reduction rate. The manufacturer, as the follower, chooses its carbon emission reduction rate based on the leader's decision.

The parameters and decision variables involved in this paper are shown in Table 1.

Table1. Parameters and decision variables

\begin{tabular}{ll}
\hline Parameters & Parameter Definitions \\
\hline$w$ & Wholesales price of per unit product of the manufacturer \\
$p$ & Sales price of per unit product of the retailer \\
$\pi_{m}, \pi_{r}, \pi$ & Production cost of per unit product of the manufacturer \\
$c_{m}, c_{r}$ & Profits of the manufacturer, the retailer and the supply chain system, respectively \\
$\gamma_{m}, \gamma_{r}$ & Carbon abatement costs of the manufacturer and the retailer, respectively \\
$D$ & Fixed demand of the market \\
$\Delta D$ & Non-fixed demand brought by costumers' low-carbon preferences \\
$\delta$ & Coefficient of demand increase brought by low-carbon preference of customers \\
$\alpha$ & Impact factor of carbon emission reduction rate of the manufacturer on demand \\
$\beta$ & Impact factor of carbon emission reduction rate of the retailer on demand \\
Decision & Decision Variable Definitions \\
Variables & Carbon emission reduction rate of the manufacturer and the retailer, respectively \\
$\theta_{m}, \theta_{r}$ &
\end{tabular}


According to the research from Wang et al. (2016) and Ji et al. (2017), there is a quadratic relationship between $C_{m}$ and $\theta_{m}$, as well as $C_{r}$ and $\theta_{r}$ :

$$
C_{m}=\frac{1}{2} \gamma_{m} \theta_{m}^{2}, \quad C_{r}=\frac{1}{2} \gamma_{r} \theta_{r}^{2}
$$

where $\theta_{m}=\theta_{m}^{\prime} \times 100, \theta_{r}=\theta_{r}^{\prime} \times 100, \theta_{m}^{\prime}$ and $\theta_{r}^{\prime}$ are the ratios and satisfy $0<\theta_{m}^{\prime}, \theta_{r}^{\prime}<1 ; \gamma_{m}$ and $\gamma_{r}$ represent the extent of the difficulty of carbon emission reduction. For the purpose of simplification, we assume $\gamma_{m}=\gamma_{r}=\gamma>0$. The above quadratic function indicates that the carbon abatement cost increases quadratically with the carbon emission reduction rate. The larger $\theta_{m}$ and $\theta_{r}$, the better the carbon emission reduction effect, correspondingly, the higher $C_{m}$ and $C_{r}$.

We suppose that consumers have a low-carbon preference, so the demand for products consists of two parts: the original fixed demand $D$ and the change demand $\Delta D . \Delta D$ is influenced by $\theta_{m}$ and $\theta_{r}$. To simplify the calculation, we assume $D=0$. According to Li et al. (2014), the demand function for low carbon products is as followed:

$$
\Delta D=\delta \theta_{m}^{\alpha} \theta_{r}^{\beta}
$$

where $\delta, \alpha$ and $\beta$ are greater than zero. It is generally believed that $\alpha+\beta<1$ which will ensure the diseconomies of scale, that is, continuously increasing carbon emission reduction rate does not guarantee continuously increasing demand of low-carbon products.

On the basis of the analysis above, we can establish the profit functions for the manufacturer, the retailer and the entire supply chain system:

The revenue of the manufacturer is wholesales income $w(D+\Delta D)$. The costs of the manufacturer consist of production cost $c(D+\Delta D)(w>c)$ and carbon abatement cost $C_{m}$. Therefore, the profit function of the manufacturer can be induced as follows.

$$
\Pi_{m}=(w-c)(D+\Delta D)-C_{m}=(w-c) \delta \theta_{m}^{\alpha} \theta_{r}^{\beta}-\frac{1}{2} \gamma \theta_{m}^{2}
$$

The revenue of the retailer is sales income $p(D+\Delta D)$. The costs of the retailer include procurement cost of products $w(D+\Delta D)(p>w)$ and carbon abatement cost $C_{r}$. Thus, the retailer's profit function is as follows.

$$
\Pi_{r}=(p-w)(D+\Delta D)-C_{r}=(p-w) \delta \theta_{m}^{\alpha} \theta_{r}^{\beta}-\frac{1}{2} \gamma \theta_{r}^{2}
$$

The total profit of the entire supply chain system is equal to the sum of the profits of the manufacturer and the retailer, that is,

$$
\Pi=\Pi_{m}+\Pi_{r}=\delta \theta_{m}^{\alpha} \theta_{r}^{\beta}(p-c)-\frac{1}{2} \gamma\left(\theta_{m}^{2}+\theta_{r}^{2}\right)
$$

\section{Model Construction and Solution}

\subsection{Nash Game Model (N model)}

In $\mathrm{N}$ model, the manufacturer and the retailer almost have the same bargaining power, so they simultaneously make decisions on the optimal carbon emission reduction to maximize their own profits. This question can be described as follows.

$$
\begin{aligned}
& \max _{\theta_{m 1}} \Pi_{m 1}=(w-c) \delta \theta_{m 1}^{\alpha} \theta_{r 1}^{\beta}-\frac{1}{2} \gamma \theta_{m 1}^{2} \\
& \max _{\theta_{r 1}} \Pi_{r 1}=(p-w) \delta \theta_{m 1}^{\alpha} \theta_{r 1}^{\beta}-\frac{1}{2} \gamma \theta_{r 1}^{2}
\end{aligned}
$$

The first derivatives of $\Pi_{m 1}$ and $\Pi_{r 1}$ on $\theta_{m 1}$ and $\theta_{r 1}$, respectively, are given by

$$
\begin{aligned}
& \frac{d \Pi_{m 1}}{d \theta_{m 1}}=\alpha(w-c) \delta \theta_{m 1}^{\alpha-1} \theta_{r 1}^{\beta}-\gamma \theta_{m 1}=0 \\
& \frac{d \Pi_{r 1}}{d \theta_{r 1}}=\beta(p-w) \delta \theta_{m 1}^{\alpha} \theta_{r 1}^{\beta-1}-\gamma \theta_{r 1}=0
\end{aligned}
$$

Then the second derivatives are as follows, respectively. 


$$
\begin{aligned}
\frac{d^{2} \Pi_{m 1}}{d \theta_{m 1}^{2}} & =\alpha(\alpha-1)(w-c) \delta \theta_{m 1}^{\alpha-2} \theta_{r 1}^{\beta}-\gamma<0 \\
\frac{d^{2} \Pi_{r 1}}{d \theta_{r 1}^{2}} & =\beta(\beta-1)(p-w) \delta \theta_{m 1}^{\alpha} \theta_{r 1}^{\beta-2}-\gamma<0
\end{aligned}
$$

where $\alpha+\beta<1$.

Therefore, $\Pi_{m 1}$ and $\Pi_{r 1}$ are both strictly concave functions, which implies that $\theta_{m 1}^{*}$ and $\theta_{r 1}^{*}$ are unique to maximize the profits of the manufacturer and the retailer, respectively. Combining eqs (4) and (5), we can obtain the optimal solutions $\theta_{m 1}^{*}$ and $\theta_{r 1}^{*}$.

$$
\begin{gathered}
\left\{\begin{array}{l}
\alpha(w-c) \delta \theta_{m 1}^{\alpha-1} \theta_{r 1}^{\beta}-\gamma \theta_{m 1}=0 \\
\beta(p-w) \delta \theta_{m 1}^{\alpha} \theta_{r 1}^{\beta-1}-\gamma \theta_{r 1}=0
\end{array}\right. \\
\left\{\begin{array}{l}
\theta_{m 1}^{*}=\left(\frac{\delta}{\gamma}\right)^{\frac{1}{2-\alpha-\beta}}[\beta(p-w)]^{\frac{\beta}{2(2-\alpha-\beta)}}[\alpha(w-c)]^{\frac{2-\beta}{2(2-\alpha-\beta)}} \\
\theta_{r 1}^{*}=\left(\frac{\delta}{\gamma}\right)^{\frac{1}{2-\alpha-\beta}}[\beta(p-w)]^{\frac{2-\alpha}{2(2-\alpha-\beta)}}[\alpha(w-c)]^{\frac{\alpha}{2(2-\alpha-\beta)}}
\end{array}\right.
\end{gathered}
$$

Thus, the optimal profits of the manufacturer and the retailer are as follows, respectively.

$$
\begin{aligned}
& \Pi_{m 1}^{*}=\left(\frac{1}{\alpha}-\frac{1}{2}\right) \delta^{\frac{2}{2-\alpha-\beta}}\left(\frac{1}{\gamma}\right)^{\frac{\alpha+\beta}{2-\alpha-\beta}}[\beta(p-w)]^{\frac{\beta}{2-\alpha-\beta}}[\alpha(w-c)]^{\frac{2-\beta}{2-\alpha-\beta}} \\
& \Pi_{r 1}^{*}=\left(\frac{1}{\beta}-\frac{1}{2}\right) \delta^{\frac{2}{2-\alpha-\beta}}\left(\frac{1}{\gamma}\right)^{\frac{\alpha+\beta}{2-\alpha-\beta}}[\beta(p-w)]^{\frac{2-\alpha}{2-\alpha-\beta}}[\alpha(w-c)]^{\frac{\alpha}{2-\alpha-\beta}}
\end{aligned}
$$

Substituting into equation (3), we can obtain the optimal profit of the supply chain system.

$$
\Pi_{1}^{*}=\delta^{\frac{2}{2-\alpha-\beta}}\left(\frac{1}{\gamma}\right)^{\frac{\alpha+\beta}{2-\alpha-\beta}}[\beta(p-w)]^{\frac{\beta}{2-\alpha-\beta}}[\alpha(w-c)]^{\frac{\alpha}{2-\alpha-\beta}}\left[\left(1-\frac{1}{2} \alpha\right)(w-c)+\left(1-\frac{1}{2} \beta\right)(p-w)\right]
$$

\subsection{Manufacturer Stackelberg Game Model (M model)}

In M model, the manufacturer is the leader, and the retailer is the follower, which suggests that the bargaining power of the manufacturer is stronger than that of the retailer. In many industries, Monopolistic manufacturers, such as Dell, Sony, and Lenovo, possess more bargaining power than some of their retailers. Thus, the manufacturer first determines its optimal carbon emission reduction rate and then the retailer makes its optimal decision, in order to maximize their respective profits. This is a case of backward induction where the manufacturer's emission reduction rate is first given and then the retailer makes decision.

The first derivative of $\Pi_{r 2}$ on $\theta_{r 2}$ is given by

$$
\max _{\theta_{r 2}} \Pi_{r 2}=(p-w) \delta \theta_{m 2}^{\alpha} \theta_{r 2}^{\beta}-\frac{1}{2} \gamma \theta_{r 2}^{2}
$$

$$
\frac{d \Pi_{r 2}}{d \theta_{r 2}}=\beta(p-w) \delta \theta_{m 2}^{\alpha} \theta_{r 2}^{\beta-1}-\gamma \theta_{r 2}=0
$$

Then the second derivative is as follows.

$$
\frac{d^{2} \Pi_{r 2}}{d \theta_{r 2}^{2}}=\beta(\beta-1)(p-w) \delta \theta_{m 2}^{\alpha} \theta_{r 2}^{\beta-2}-\gamma<0
$$

The profit function of the retailer is a strictly concave function, so there is a unique optimal carbon emission reduction ratio of the retailer $\theta_{r 2}^{*}$ maximizing its profit $\Pi_{r 2}$. Solving eq (6), we can obtain $\theta_{r 2}^{*}$. 


$$
\theta_{r 2}^{*}=\left(\frac{\delta}{\gamma}\right)^{\frac{1}{2-\beta}}[\beta(p-w)]^{\frac{1}{2-\beta}} \theta_{m 2}^{\frac{\alpha}{2-\beta}}
$$

Substituting eq (7) into eq (1), we can obtain the profit function of the manufacturer.

$$
\Pi_{m 2}=(w-c) \delta^{\frac{2}{2-\beta}}\left(\frac{1}{\gamma}\right)^{\frac{\beta}{2-\beta}}[\beta(p-w)]^{\frac{\beta}{2-\beta}} \theta_{m 2}^{\frac{2 \alpha}{2-\beta}}-\frac{1}{2} \gamma \theta_{m 2}^{2}
$$

The first derivative of $\Pi_{m 2}$ on $\theta_{m 2}$ is as follows.

$$
\frac{d \Pi_{m 2}}{d \theta_{m 2}}=(w-c) \delta^{\frac{2}{2-\beta}}\left(\frac{1}{\gamma}\right)^{\frac{\beta}{2-\beta}} \frac{2 \alpha}{2-\beta}[\beta(p-w)]^{\frac{\beta}{2-\beta}} \theta_{m 2}^{\frac{2 \alpha}{2-\beta}-1}-\gamma \theta_{m 2}=0
$$

Then the second derivative is obtained.

$$
\frac{d^{2} \Pi_{m 2}}{d \theta_{m 2}^{2}}=(w-c) \delta^{\frac{2}{2-\beta}}\left(\frac{1}{\gamma}\right)^{\frac{\beta}{2-\beta}} \frac{2 \alpha}{2-\beta}\left(\frac{2 \alpha}{2-\beta}-1\right)[\beta(p-w)]^{\frac{\beta}{2-\beta}} \theta_{m 2}^{\frac{2 \alpha}{2-\beta}-2}-\gamma<0
$$

The profit function of the manufacturer is a strictly concave function, so there is a unique $\theta_{m 2}^{*}$ to maximize $\Pi_{m 2}$. Solving eq (8), we can obtain the optimal carbon emission reduction rate of the manufacturer.

$$
\theta_{m 2}^{*}=\left(\frac{\delta}{\gamma}\right)^{\frac{1}{2-\alpha-\beta}}\left(\frac{2}{2-\beta}\right)^{\frac{2-\beta}{2(2-\alpha-\beta)}}[\beta(p-w)]^{\frac{\beta}{2(2-\alpha-\beta)}}[\alpha(w-c)]^{\frac{2-\beta}{2(2-\alpha-\beta)}}
$$

By substituting eq (9) into eq (7), we can obtain the optimal carbon emission reduction rate of the retailer.

$$
\theta_{r 2}^{*}=\left(\frac{\delta}{\gamma}\right)^{\frac{1}{2-\alpha-\beta}}\left(\frac{2}{2-\beta}\right)^{\frac{\alpha}{2(2-\alpha-\beta)}}[\beta(p-w)]^{\frac{2-\alpha}{2(2-\alpha-\beta)}}[\alpha(w-c)]^{\frac{\alpha}{2(2-\alpha-\beta)}}
$$

Then the optimal profits of the manufacturer and the retailer are as follows.

$$
\begin{gathered}
\Pi_{m 2}^{*}=\left(\frac{1}{\alpha}-\frac{1}{2-\beta}\right) \delta^{\frac{2}{2-\alpha-\beta}}\left(\frac{1}{\gamma}\right)^{\frac{\alpha+\beta}{2-\alpha-\beta}}\left(\frac{2}{2-\beta}\right)^{\frac{\alpha}{2-\alpha-\beta}}[\beta(p-w)]^{\frac{\beta}{2-\alpha-\beta}}[\alpha(w-c)]^{\frac{2-\beta}{2-\alpha-\beta}} \\
\Pi_{r 2}^{*}=\left(\frac{1}{\beta}-\frac{1}{2}\right) \delta^{\frac{2}{2-\alpha-\beta}}\left(\frac{1}{\gamma}\right)^{\frac{\alpha+\beta}{2-\alpha-\beta}}\left(\frac{2}{2-\beta}\right)^{\frac{\alpha}{2-\alpha-\beta}}[\beta(p-w)]^{\frac{2-\alpha}{2-\alpha-\beta}}[\alpha(w-c)]^{\frac{\alpha}{2-\alpha-\beta}}
\end{gathered}
$$

The optimal total profit of the supply chain system is:

$$
\Pi_{2}^{*}=\delta^{\frac{2}{2-\alpha-\beta}}\left(\frac{1}{\gamma}\right)^{\frac{\alpha+\beta}{2-\alpha-\beta}}\left(\frac{2}{2-\beta}\right)^{\frac{\alpha}{2-\alpha-\beta}}[\beta(p-w)]^{\frac{\beta}{2-\alpha-\beta}}[\alpha(w-c)]^{\frac{\alpha}{2-\alpha-\beta}}\left[\left(1-\frac{\alpha}{2-\beta}\right)(w-c)+\left(1-\frac{1}{2} \beta\right)(p-w)\right]
$$

\subsection{Retailer Stackelberg Game Model (R model)}

In $\mathrm{R}$ model, the retailer is the leader, and the manufacturer is the follower, suggesting that the bargaining power of the retailer is stronger than that of the manufacturer, which is opposite with $\mathrm{M}$ model. Many large retailers, such as Wal-Mart and Amazon, have stronger bargaining power than some of their upstream enterprises. Thus, the retailer first determines its optimal carbon emission reduction rate and then the manufacturer makes its optimal decision, in order to maximize their respective profits. This is also a case of backward induction where the retailer's emission reduction rate is first given and then the manufacturer makes decision.

$$
\max _{\theta_{m 3}} \Pi_{m 3}=(w-c) \delta \theta_{m 3}^{\alpha} \theta_{r 3}^{\beta}-\frac{1}{2} \gamma \theta_{m 3}^{2}
$$

The first derivative of $\Pi_{m 3}$ on $\theta_{m 3}$ is given by 


$$
\frac{d \Pi_{m 3}}{d \theta_{m 3}}=\alpha(w-c) \delta \theta_{m 3}^{\alpha-1} \theta_{r 3}^{\beta}-\gamma \theta_{m 3}=0
$$

Then the second derivative is as follows.

$$
\frac{d^{2} \Pi_{m 3}}{d \theta_{m 3}^{2}}=\alpha(\alpha-1)(w-c) \delta \theta_{m 3}^{\alpha-2} \theta_{r 3}^{\beta}-\gamma<0
$$

$\Pi_{m 3}$ is a strictly concave function, so there is a unique $\theta_{m 3}^{*}$ maximizing $\Pi_{m 3}$. Solving eq (11), we can obtain $\theta_{m 3}^{*}$.

$$
\theta_{m 3}^{*}=\left(\frac{\delta}{\gamma}\right)^{\frac{1}{2-\alpha}}[\alpha(w-c)]^{\frac{1}{2-\alpha}} \theta_{r 3}^{\frac{\beta}{2-\alpha}}
$$

Substituting eq (12) into eq (2), we can obtain the profit function of the retailer.

$$
\Pi_{r 3}=(p-w) \delta^{\frac{2}{2-\alpha}}\left(\frac{1}{\gamma}\right)^{\frac{\alpha}{2-\alpha}}[\alpha(w-c)]^{\frac{\alpha}{2-\alpha}} \theta_{r 3}^{\frac{2 \beta}{2-\alpha}}-\frac{1}{2} \gamma \theta_{r 3}^{2}
$$

The first derivative of $\Pi_{r 3}$ on $\theta_{r 3}$ is as follows.

$$
\frac{d \Pi_{r 3}}{d \theta_{r 3}}=(p-w) \delta^{\frac{2}{2-\alpha}}\left(\frac{1}{\gamma}\right)^{\frac{\alpha}{2-\alpha}} \frac{2 \beta}{2-\alpha}[\alpha(w-c)]^{\frac{\alpha}{2-\alpha}} \theta_{r 3}^{\frac{2 \beta}{2-\alpha}-1}-\gamma \theta_{r 3}
$$

Then the second derivative is obtained.

$$
\frac{d^{2} \Pi_{r 3}}{d \theta_{r 3}^{2}}=(p-w) \delta^{\frac{2}{2-\alpha}}\left(\frac{1}{\gamma}\right)^{\frac{\alpha}{2-\alpha}} \frac{2 \beta}{2-\alpha}\left(\frac{2 \beta}{2-\alpha}-1\right)[\alpha(w-c)]^{\frac{\alpha}{2-\alpha}} \theta_{r 3}^{\frac{2 \beta}{2-\alpha}-2}-\gamma<0
$$

$\Pi_{r 3}$ is a strictly concave function, so there is a unique $\theta_{r 3}^{*}$ maximizing $\Pi_{r 3}$. Solving eq (13), we can obtain $\theta_{r 3}^{*}$.

$$
\theta_{r 3}^{*}=\left(\frac{\delta}{\gamma}\right)^{\frac{1}{2-\alpha-\beta}}\left(\frac{2}{2-\alpha}\right)^{\frac{2-\alpha}{2(2-\alpha-\beta)}}[\beta(p-w)]^{\frac{2-\alpha}{2(2-\alpha-\beta)}}[\alpha(w-c)]^{\frac{\alpha}{2(2-\alpha-\beta)}}
$$

Substituting eq (14) into eq (12), we can obtain $\theta_{m 3}^{*}$.

$$
\theta_{m 3}^{*}=\left(\frac{\delta}{\gamma}\right)^{\frac{1}{2-\alpha-\beta}}\left(\frac{2}{2-\alpha}\right)^{\frac{\beta}{2(2-\alpha-\beta)}}[\beta(p-w)]^{\frac{\beta}{2(2-\alpha-\beta)}}[\alpha(w-c)]^{\frac{2-\beta}{2(2-\alpha-\beta)}}
$$

Then the optimal profits of the manufacturer and the retailer are:

$$
\begin{gathered}
\Pi_{m 3}^{*}=\left(\frac{1}{\alpha}-\frac{1}{2}\right) \delta^{\frac{2}{2-\alpha-\beta}}\left(\frac{1}{\gamma}\right)^{\frac{\alpha+\beta}{2-\alpha-\beta}}\left(\frac{2}{2-\alpha}\right)^{\frac{\beta}{2-\alpha-\beta}}[\beta(p-w)]^{\frac{\beta}{2-\alpha-\beta}}[\alpha(w-c)]^{\frac{2-\beta}{2-\alpha-\beta}} \\
\Pi_{r 3}^{*}=\left(\frac{1}{\beta}-\frac{1}{2-\alpha}\right) \delta^{\frac{1}{2-\alpha-\beta}}\left(\frac{1}{\gamma}\right)^{\frac{\alpha+\beta}{2-\alpha-\beta}}\left(\frac{2}{2-\alpha}\right)^{\frac{\beta}{2-\alpha-\beta}}[\beta(p-w)]^{\frac{2-\alpha}{2-\alpha-\beta}}[\alpha(w-c)]^{\frac{\alpha}{2-\alpha-\beta}}
\end{gathered}
$$

The optimal total profit of the supply chain system is as follows.

$$
\Pi_{3}^{*}=\delta^{\frac{1}{2-\alpha-\beta}}\left(\frac{1}{\gamma}\right)^{\frac{\alpha+\beta}{2-\alpha-\beta}}\left(\frac{2}{2-\alpha}\right)^{\frac{\beta}{2-\alpha-\beta}}[\beta(p-w)]^{\frac{\beta}{2-\alpha-\beta}}[\alpha(w-c)]^{\frac{\alpha}{2-\alpha-\beta}}\left[\left(1-\frac{1}{2} \alpha\right)(w-c)+\left(1-\frac{\beta}{2-\alpha}\right)(p-w)\right]
$$

\section{Comparative Analysis of Solutions between Different Game Models}

The optimal carbon emission reduction decisions and optimal profits of the manufacturer and the retailer in 
different game models are obtained and shown in Table 2. It is difficult to directly compare the optimal decisions and profits of supply chain enterprises in different game models. We use numerical simulations to analyze. Assume that initial values of the parameters are as follows: $p=17, w=10, c=5, \alpha=0.15, \beta=0.2$, $\delta=10, \gamma=5$. According to Table 2, it is not difficult to find that $\alpha$ and $\beta$ are the determinants of the differences in the optimal decisions and profits among different game models. Therefore, the differences of the carbon emission reduction decisions and profits of three game models are analyzed under the change of $\alpha$ and $\beta$, respectively.

Table 2. The optimal solutions in three game models

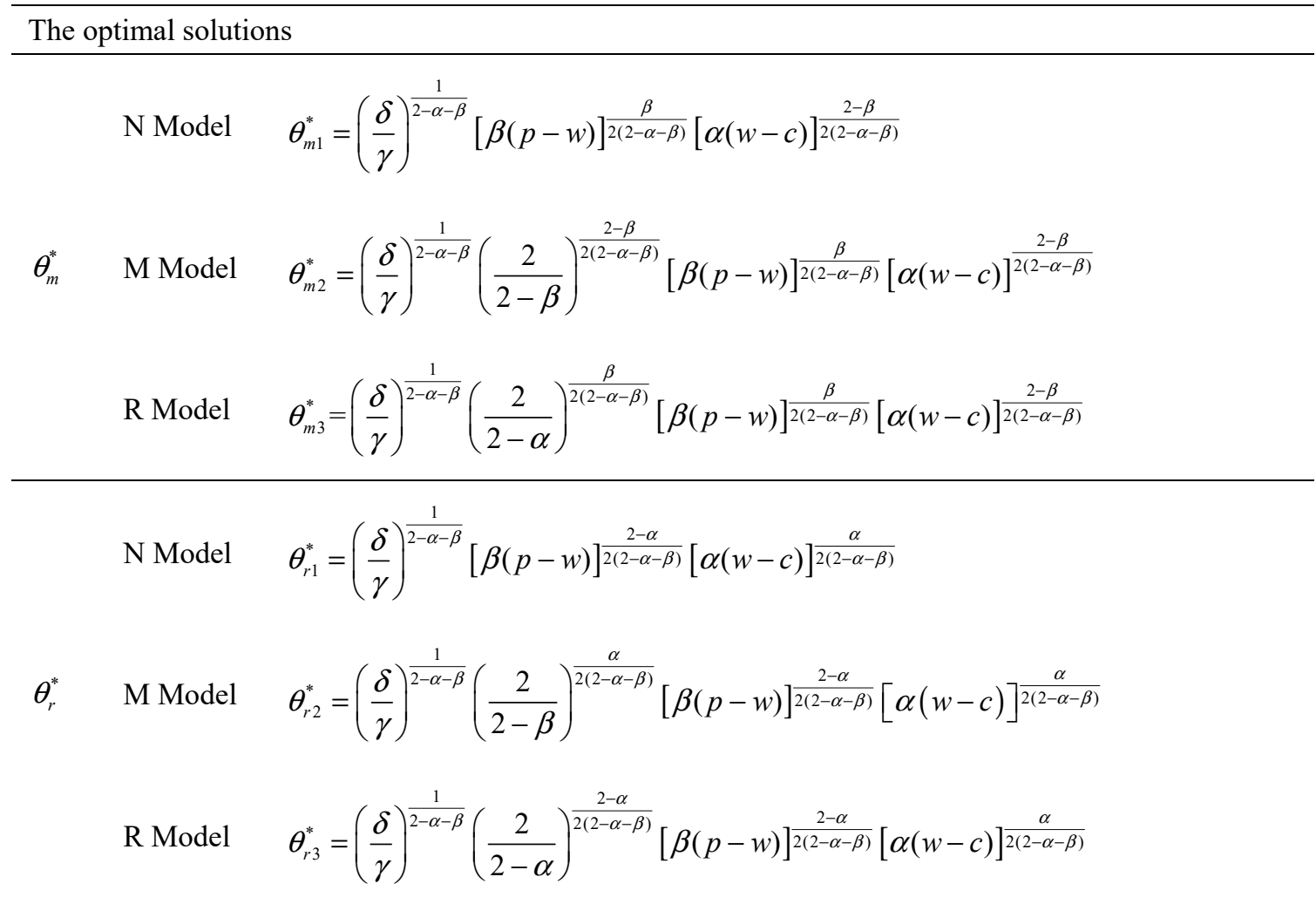

$\mathrm{N}$ Model $\quad \Pi_{m 1}^{*}=\left(\frac{1}{\alpha}-\frac{1}{2}\right) \delta^{\frac{2}{2-\alpha-\beta}}\left(\frac{1}{\gamma}\right)^{\frac{\alpha+\beta}{2-\alpha-\beta}}[\beta(p-w)]^{\frac{\beta}{2-\alpha-\beta}}[\alpha(w-c)]^{\frac{2-\beta}{2-\alpha-\beta}}$

$\Pi_{m}^{*} \quad$ M Model $\quad \Pi_{m 2}^{*}=\left(\frac{1}{\alpha}-\frac{1}{2-\beta}\right) \delta^{\frac{2}{2-\alpha-\beta}}\left(\frac{1}{\gamma}\right)^{\frac{\alpha+\beta}{2-\alpha-\beta}}\left(\frac{2}{2-\beta}\right)^{\frac{\alpha}{2-\alpha-\beta}}[\beta(p-w)]^{\frac{\beta}{2-\alpha-\beta}}[\alpha(w-c)]^{\frac{2-\beta}{2-\alpha-\beta}}$

R Model $\quad \Pi_{m 3}^{*}=\left(\frac{1}{\alpha}-\frac{1}{2}\right) \delta^{\frac{2}{2-\alpha-\beta}}\left(\frac{1}{\gamma}\right)^{\frac{\alpha+\beta}{2-\alpha-\beta}}\left(\frac{2}{2-\alpha}\right)^{\frac{\beta}{2-\alpha-\beta}}[\beta(p-w)]^{\frac{\beta}{2-\alpha-\beta}}[\alpha(w-c)]^{\frac{2-\beta}{2-\alpha-\beta}}$

$\mathrm{N}$ Model $\quad \Pi_{r 1}^{*}=\left(\frac{1}{\beta}-\frac{1}{2}\right) \delta^{\frac{2}{2-\alpha-\beta}}\left(\frac{1}{\gamma}\right)^{\frac{\alpha+\beta}{2-\alpha-\beta}}[\beta(p-w)]^{\frac{2-\alpha}{2-\alpha-\beta}}[\alpha(w-c)]^{\frac{\alpha}{2-\alpha-\beta}}$

$\Pi_{r}^{*}$

M Model $\quad \Pi_{r 2}^{*}=\left(\frac{1}{\beta}-\frac{1}{2}\right) \delta^{\frac{2}{2-\alpha-\beta}}\left(\frac{1}{\gamma}\right)^{\frac{\alpha+\beta}{2-\alpha-\beta}}\left(\frac{2}{2-\beta}\right)^{\frac{\alpha}{2-\alpha-\beta}}[\beta(p-w)]^{\frac{2-\alpha}{2-\alpha-\beta}}[\alpha(w-c)]^{\frac{\alpha}{2-\alpha-\beta}}$ 
R Model $\quad \Pi_{r 3}^{*}=\left(\frac{1}{\beta}-\frac{1}{2-\alpha}\right) \delta^{\frac{1}{2-\alpha-\beta}}\left(\frac{1}{\gamma}\right)^{\frac{\alpha+\beta}{2-\alpha-\beta}}\left(\frac{2}{2-\alpha}\right)^{\frac{\beta}{2-\alpha-\beta}}[\beta(p-w)]^{\frac{2-\alpha}{2-\alpha-\beta}}[\alpha(w-c)]^{\frac{\alpha}{2-\alpha-\beta}}$

N Model

$$
\Pi_{1}^{*}=\delta^{\frac{2}{2-\alpha-\beta}}\left(\frac{1}{\gamma}\right)^{\frac{\alpha+\beta}{2-\alpha-\beta}}[\beta(p-w)]^{\frac{\beta}{2-\alpha-\beta}}[\alpha(w-c)]^{\frac{\alpha}{2-\alpha-\beta}}
$$

$\Pi^{*} \quad$ M Model

$$
\begin{aligned}
& {\left[\left(1-\frac{1}{2} \alpha\right)(w-c)+\left(1-\frac{1}{2} \beta\right)(p-w)\right]} \\
& \Pi_{2}^{*}=\delta^{\frac{2}{2-\alpha-\beta}}\left(\frac{1}{\gamma}\right)^{\frac{\alpha+\beta}{2-\alpha-\beta}}\left(\frac{2}{2-\beta}\right)^{\frac{\alpha}{2-\alpha-\beta}}[\beta(p-w)]^{\frac{\beta}{2-\alpha-\beta}}[\alpha(w-c)]^{\frac{\alpha}{2-\alpha-\beta}}
\end{aligned}
$$

$$
\left[\left(1-\frac{\alpha}{2-\beta}\right)(w-c)+\left(1-\frac{1}{2} \beta\right)(p-w)\right]
$$

$$
\Pi_{3}^{*}=\delta^{\frac{1}{2-\alpha-\beta}}\left(\frac{1}{\gamma}\right)^{\frac{\alpha+\beta}{2-\alpha-\beta}}\left(\frac{2}{2-\alpha}\right)^{\frac{\beta}{2-\alpha-\beta}}[\beta(p-w)]^{\frac{\beta}{2-\alpha-\beta}}[\alpha(w-c)]^{\frac{\alpha}{2-\alpha-\beta}}
$$

R Model

$$
\left[\left(1-\frac{1}{2} \alpha\right)(w-c)+\left(1-\frac{\beta}{2-\alpha}\right)(p-w)\right]
$$

\subsection{Influence of $\alpha$}

Due to $0<\alpha+\beta<1$, let $\alpha$ be taken from 0.1-0.8, with a step size of 0.1 . Under the fluctuation of $\alpha$, the impact status of different game models on the optimal decisions of supply chain enterprises is shown in Figure 1-2. Figure 1-2 illustrates that with the increase of $\alpha$, the differences in the carbon emission reduction rates and profits of supply chain enterprises between different game models are all increasing. And for carbon emission reduction rates, except for $\theta_{m 3}^{*}-\theta_{m 2}^{*}<0$, the others are all greater than 0 ; for profits, except for $\Pi_{r 3}^{*}-\Pi_{r 2}^{*}<0$ and $\Pi_{3}^{*}-\Pi_{2}^{*}<0$, the others are all greater than 0 .

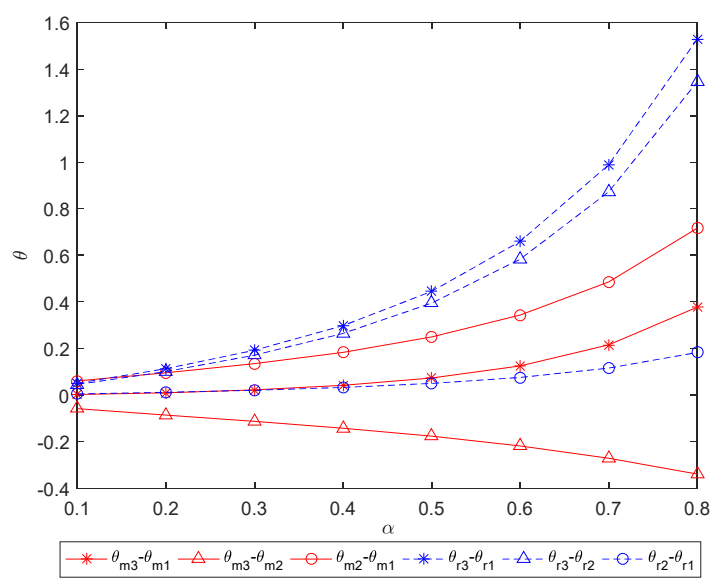

Figure 1. Differences in carbon emission reduction rates of different game models under $\alpha$ fluctuations 


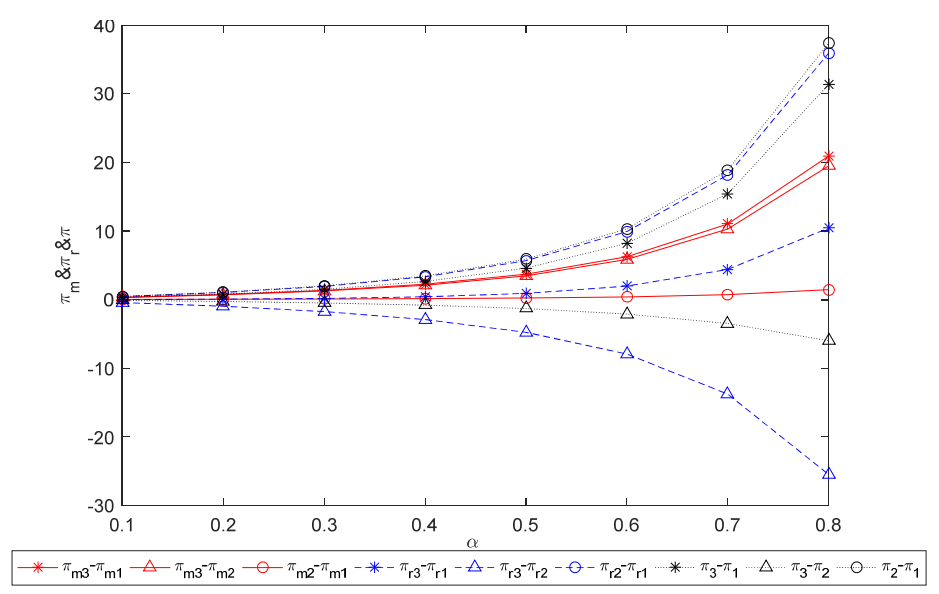

Figure 2. Differences in profits of different game models under $\alpha$ fluctuations

\subsection{Influence of $\beta$}

Due to $0<\alpha+\beta<1$, we suppose $\beta=0.1-0.8$ and per step size is 0.1 . As $\beta$ increases, the impact status of different game models on the optimal decisions of supply chain enterprises is shown in the following figures, and the conclusion is consistent with the case of $\alpha$.

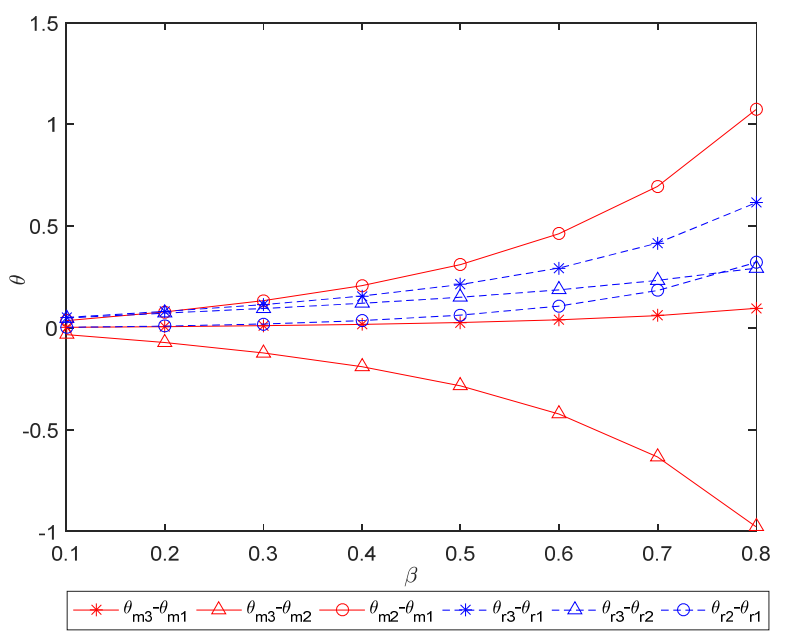

Figure 3. Differences in carbon emission reduction rates of different game models under $\beta$ fluctuations

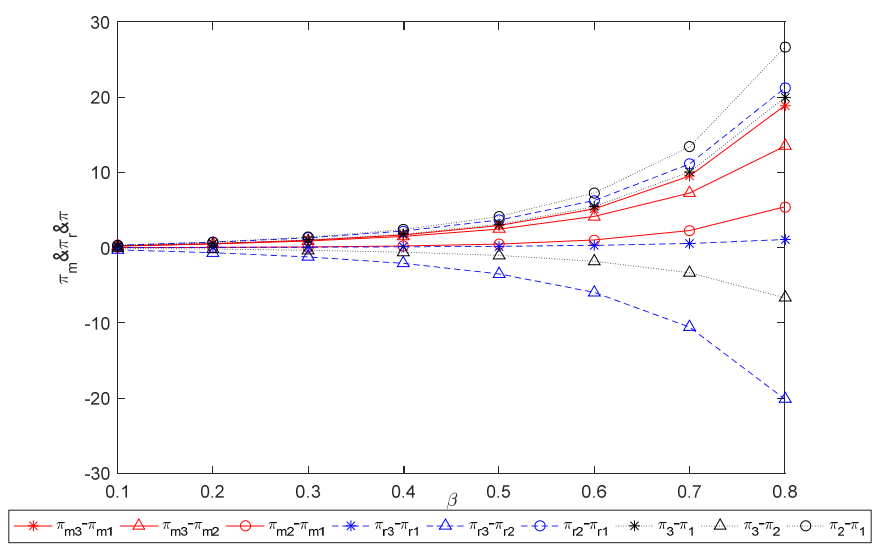

Figure 4. Differences in profits of different game models under $\beta$ fluctuations

\section{Results Discussion}

According to the above simulation analysis, we can easily reach the following conclusions: 
Proposition $1 \theta_{m 1}^{*}<\theta_{m 3}^{*}<\theta_{m 2}^{*} ; \theta_{r 1}^{*}<\theta_{r 2}^{*}<\theta_{r 3}^{*}$.

Proposition 1 indicates that the optimal carbon emission reduction rates of the manufacturer and the retailer in $\mathrm{N}$ model are always less than that in $\mathrm{M}$ and $\mathrm{R}$ models, while the optimal carbon emission reduction rate of the manufacturer as a leader in $\mathrm{M}$ model is greater than that of the manufacturer as a follower in $\mathrm{N}$ model, and the retailer's optimal carbon emission reduction rate is the opposite, which implies that compared with Nash game, the optimal carbon emission reduction rate of supply chain enterprises in Stackelberg game is greater, and the effect is better; in the two Stackelberg games, the optimal carbon emission reduction rate as a leader is greater than that as a follower.

Proposition $2 \Pi_{m 1}^{*}<\Pi_{m 2}^{*}<\Pi_{m 3}^{*} ; \quad \Pi_{r 1}^{*}<\Pi_{r 3}^{*}<\Pi_{r 2}^{*} ; \quad \Pi_{1}^{*}<\Pi_{3}^{*}<\Pi_{2}^{*}$.

Proposition 2 indicates that the optimal profit of the manufacturer in $\mathrm{N}$ model is always less than that in $\mathrm{M}$ and $\mathrm{R}$ models, and it is the same with the retailer, and the entire supply chain system. The optimal profit of the manufacturer as a leader in $\mathrm{M}$ model is less than that of the manufacturer as a follower in $\mathrm{N}$ model and the retailer's optimal profit is the opposite. The total profit of the entire supply chain system in M model is greater than that in $\mathrm{R}$ model. According to Proposition 1, although the optimal carbon emission reduction rate of Nash game is small and its cost is low, because of the less demand it brings, its profit is always less than that in Stackelberg game. In the two Stackelberg games, as for the leader, although its demand increases, such advantage is not sufficient to make up for the cost of carbon emission reduction, which leads to its profit less than that as a follower.

\section{Conclusions}

Considering the differences in power structures between supply chain enterprises, this paper constructs Nash, Manufacturer Stackelberg, and Retailer Stackelberg game models between the manufacturer and the retailer respectively and solves the optimal carbon emission reduction decisions of the manufacturer and the retailer in these models. The differences between the optimal decisions and profits of supply chain enterprises in these models are analyzed and compared. It is concluded that compared with Nash game, Stackelberg game can not only achieve a higher level of carbon emission reduction, but also bring more profits. Therefore, supply chain enterprises should choose the optimal game strategy based on their own goals and bargaining power. On this basis, we can further consider the cooperation of carbon emission reduction between supply chains enterprises to optimize the decision of low-carbon supply chain enterprises. And the impact of government policies (e.g., carbon subsidies and carbon taxes) on the carbon emission reduction decisions of enterprises with different power structures can also be explored in the future.

\subsection{Acknowledgments}

This research was supported in part by the National Science Foundation of China (project no.:71572033).

\section{References}

Chaabane, A., Ramudhin, A., \& Paquet, M. (2011). Designing supply chains with sustainability considerations. Production Planning \& Control, 22(8), 727-741. http://dx.doi.org/10.1080/ 09537287.2010.543554

Du, S., Zhu, J., Jiao, H., \& Ye, W. (2015). Game-theoretical analysis for supply chain with consumer preference to low carbon. International Journal of Production Research, 53(12), 3753-3768. http://dx.doi.org/10.1080/00207543.2014.988888

Elhedhli, S., \& Merrick, R. (2012). Green supply chain network design to reduce carbon emissions. Transportation Research Part D: Transport and Environment, 17(5), 370-379. http://dx.doi.org/10.1016/j.trd.2012.02.002

He, L., Zhao, D., \& Xia, L. (2015). Game theoretic analysis of carbon emission abatement in fashion supply chains considering vertical incentives and channel structures. Sustainability, 7(4), 4280-4309. http://dx.doi.org/10.3390/su7044280

Hsu, C. W., Kuo, T. C., Chen, S. H., \& Hu, A. H. (2013). Using DEMATEL to develop a carbon management model of supplier selection in green supply chain management. Journal of Cleaner Production, 56, 164-172. http://dx.doi.org/10.1016/j.jclepro.2011.09.012

Hua, G., Cheng, T.C.E., \& Wang, S. (2011). Managing carbon footprints in inventory management. International Journal of Production Economics, 132(2), 178-185. http://dx.doi.org/10.1016/j.ijpe.2011.03.024

Ji, J., Zhang, Z., \& Yang, L. (2017). Carbon emission reduction decisions in the retail-/dual-channel supply chain with consumers' preference. Journal of Cleaner Production, 141, 852-867. 
http://dx.doi.org/10.1016/j.jclepro.2016.09.135

Li, Y., Zhao, D., \& Xia, L. (2014). Governmental subsidy policy for enterprises' cooperative behavior in emission reduction in low-carbon supply chain. Operations Research and Management Science, 23(4), $1-10$.

Wang, Q., Zhao, D., \& He, L. (2016). Contracting emission reduction for supply chains considering market low-carbon preference. Journal of Cleaner Production, 120, 72-84. http://dx.doi.org/10.1016/j.jclepro.2015.11.049

Yi, Y., \& Li, J. (2018). The effect of governmental policies of carbon taxes and energy-saving subsidies on enterprise decisions in a two-echelon supply chain. Journal of Cleaner Production, 181, 675-691. https://doi.org/10.1016/j.jclepro.2018.01.188

Yu, W., \& Han, R. (2017). Coordinating a two-echelon supply chain under carbon tax. Sustainability, 9(12), 2360. http://dx.doi.org/10.3390/su9122360

Zakeri, A., Dehghanian, F., Fahimnia, B., \& Sarkis, J. (2015). Carbon pricing versus emissions trading: A supply chain planning perspective. International Journal of Production Economic, 164, 197-205. http://dx.doi.org/10.1016/j.ijpe.2014.11.012

Zhao, R., Neighbour, G., Han, J., McGuirea, M., \& Deutzd, P. (2012). Using game theory to describe strategy selection for environmental risk and carbon emissions reduction in the green supply chain. Journal of Loss Prevention in the Process Industries, 25(6), 927-936. http://dx.doi.org/10.1016/j.jlp.2012.05.004

\section{Copyrights}

Copyright for this article is retained by the author(s), with first publication rights granted to the journal.

This is an open-access article distributed under the terms and conditions of the Creative Commons Attribution license (http://creativecommons.org/licenses/by/4.0/). 